\title{
Cytokines associated with necrotizing enterocolitis in extremely-low-birth-weight infants
}

\author{
Akhil Maheshwari ${ }^{1,2}$, Robert L. Schelonka ${ }^{1,3}$, Reed A. Dimmitt' ${ }^{1}$, Waldemar A. Carlo ${ }^{1}$, Breda Munoz-Hernandez ${ }^{4}$, Abhik Das ${ }^{4}$, \\ Scott A. McDonald ${ }^{4}$, Poul Thorsen ${ }^{5}$, Kristin Skogstrand ${ }^{6}$, David M. Hougaard ${ }^{6}$ and Rosemary D. Higgins ${ }^{7}$; for the Eunice Kennedy \\ Shriver National Institute of Child Health and Human Development Neonatal Research Network
}

BACKGROUND: The goal was to identify cytokines associated with necrotizing enterocolitis (NEC). Based on our earlier reports of decreased tissue expression of transforming growth factor (TGF)- $\beta$, we hypothesized that infants with NEC also have low blood TGF- $\beta$ levels. We further hypothesized that because fetal inflammation increases the risk of NEC, infants who develop NEC have elevated blood cytokine levels in early neonatal period.

METHODS: Data on 104 extremely-low-birth-weight infants with NEC and 893 without NEC from 17 centers were analyzed. Clinical information was correlated with blood cytokine levels on postnatal day 1 (D1), D3, D7, D14, and D21.

RESULTS: Male gender, non-Caucasian/non-African American ethnicity, sepsis, lower blood TGF- $\beta$ and interleukin (IL)-2 levels, and higher IL-8 levels were associated with NEC. The NEC group had lower TGF- $\beta$ levels than controls since D1. The diagnosis of NEC was associated with elevated IL-1 $\beta$, IL-6, IL-8, IL-10, monocyte chemoattractant protein-1/CC-motif ligand-2, macrophage inflammatory protein-1 $\beta / C C$-motif ligand-3, and C-reactive protein.

CONCLUSION: Clinical characteristics, such as gender and ethnicity, and low blood TGF- $\beta$ levels are associated with higher risk of NEC. Infants who developed NEC did not start with high blood levels of inflammatory cytokines, but these rose mainly after the onset of NEC.

$\mathbf{N}$ ecrotizing enterocolitis (NEC) continues to be a leading cause of morbidity and mortality in premature infants (1). Although the etiology of NEC is unclear, current evidence associates NEC with diverse pre- and postnatal factors such as placental insufficiency, chorioamnionitis, gut ischemia, altered bacterial colonization, viruses, and blood transfusions. These conditions presumably disrupt the mucosal barrier and promote translocation of luminal bacteria, which trigger an inflammatory reaction in the developing intestine (2).

Several cross-sectional studies show that NEC is associated with increased expression of inflammatory cytokines such as tumor necrosis factor, interleukin (IL)-1 $\beta$, IL-6, and IL-8/ CXC-motif ligand 8 (CXCL8) in both plasma and affected tissues (3-7). These cytokines are potential therapeutic targets in NEC because (i) preclinical evidence indicates that these cytokines can disrupt the epithelial barrier and augment intestinal injury (8) and (ii) monoclonal antibodies and/or small molecule inhibitors that can block the effect of these inflammatory mediators are now available. At the same time, concerns remain about possible harm from anticytokine therapy in preterm infants because many so-called "inflammatory" cytokines play important developmental roles in the gut mucosa and mucosa-associated immune system (5,9). Evidence from cross-sectional studies has its limitations because cytokine expression changes during normal gestational maturation and with comorbidities associated with prematurity (10). To elucidate the pathophysiological role of cytokines in NEC, there is a need for longitudinal comparison of cytokine concentrations before and after onset of NEC and in infants who eventually developed NEC vs. others who did not. Toward this goal, we performed a secondary analysis of data obtained as part of the Eunice Kennedy Shriver National Institute of Child Health and Human Development Neonatal Research Network Cytokine Study, a prospective multicenter study in which extremelylow-birth-weight (ELBW) infants were enrolled and clinical information and serial cytokine measurements were collected from birth through postnatal day 21 (D21) (11).

We have shown recently that premature infants may be at risk of NEC due to a developmental deficiency of transforming growth factor- $\beta$ (TGF- $\beta$ ) in the intestine, which is further accentuated during NEC (12). In this study, we hypothesized that decreased tissue expression of TGF- $\beta$ is a systemic phenomenon reflected in blood samples from patients who develop NEC. In addition, in view of the epidemiological association of NEC with fetal inflammation related to chorioamnionitis, prolonged rupture of membranes, and Ureaplasma infections $(13,14)$, we hypothesized that infants who develop NEC have elevated serum cytokine levels in early neonatal period and before the onset of NEC.

'Department of Pediatrics, University of Alabama at Birmingham, Birmingham, Alabama; ${ }^{2}$ Department of Pediatrics, University of Illinois at Chicago, Chicago, Illinois;

${ }^{3}$ Department of Pediatrics, Oregon Health and Science University, Portland, Oregon; ${ }^{4}$ Statistics and Epidemiology Unit, RTI International, Research Triangle Park, North Carolina; ${ }^{5}$ Department of Obstetrics and Gynecology, Lillebaelt Hospital, Kolding, Denmark; ${ }^{6}$ Department of Clinical Biochemistry and Immunology, Statens Serum Institut, Copenhagen, Denmark; ${ }^{7}$ Eunice Kennedy Shriver National Institute of Child Health and Human Development, National Institutes of Health, Bethesda, Maryland. Correspondence: Akhil Maheshwari (akhil1@uic.edu) 


\section{RESULTS}

\section{Patient Characteristics}

A total of 1,067 infants with birth weights between 410 and $1,000 \mathrm{~g}$ were admitted to the participating centers during the study period and were enrolled in the study within $72 \mathrm{~h}$. In the final analysis, 997 infants were included; 70 neonates were excluded due to death in the first $7 \mathrm{~d}(n=43)$, birth defects $(n=13)$, or early-onset sepsis $(n=14)$.

NEC was recorded in 104 of 997 (10.4\%) infants at a median age of $23.5 \mathrm{~d}$ (range: 1-114 d). The distribution of gestational age, birth weight, and gender was similar in NEC and control groups (Table 1 ). The NEC group had a higher percentage of African-American infants $(61 / 104,58.6 \%$ in the NEC group vs. $423 / 997,47.4 \%$ controls; $P=0.02)$ and also had a higher incidence of sepsis ( 52.9 vs. $41.6 \% ; P=0.02$ ).

\section{Longitudinal Changes in Blood Cytokines}

All cytokines showed significant time trends $(P<0.05)$ during the first 3 postnatal weeks. IL-1 $\beta$, IL-2, IL-4, IL-5, IL-6, IL-10, IL-12, IL-17, lymphotoxin- $\alpha$, granulocyte macrophage colony-stimulating factor, and neurotrophin-4 (NT-4) showed a decreasing trend over time, whereas IL-18, brain-derived neurotrophic factor, macrophage inflammatory protein (MIP)-1 $\beta / \mathrm{CC}$-motif ligand-4 (CCL4), matrix metalloproteinase-9, regulated on activation, normal T-cell expressed and secreted (RANTES)/CCL5, TGF- $\beta_{1}$, and C-reactive protein (CRP) increased with postnatal age. Infants in the NEC group had lower TGF- $\beta$ levels during the study period than controls (median: 1871, range: $467-11,431 \mathrm{pg} / \mathrm{ml}$ vs. 2,501, range: $202-12,750 \mathrm{pg} / \mathrm{ml} ; P<0.05)$. There was also a trend toward lower IL-2 concentrations in the NEC group (median: 58, range: $3-430 \mathrm{pg} / \mathrm{ml}$ vs. 70 , range: $3-3,118 \mathrm{pg} / \mathrm{ml} ; P=$ 0.07). In contrast, IL-8/CXCL8 levels were higher in the NEC group than controls (median: 4,527, range: $297-837,973 \mathrm{pg}$ /

Table 1. Demographic and clinical information

\begin{tabular}{lcc}
\hline Variable name & NEC group & Controls $^{\mathrm{a}}$ \\
\hline Total cases & 104 & 893 \\
Birth weight (mean \pm SD) & $750.2 \pm 137.4$ & $766.9 \pm 140.3$ \\
Gestational age (wk; mean \pm SD) & $25.93 \pm 1.9$ & $25.64 \pm 1.6$ \\
Gender (\% male) & 52.9 & 47.4 \\
Ethnicity & & \\
$\quad$ African American (\%) & $58.6^{\mathrm{b}}$ & $47.4^{\mathrm{b}}$ \\
$\quad$ White (\%) & 24.0 & 31.7 \\
$\quad$ Others (\%) & 17.3 & 20.9 \\
Tocolytics & 48.5 & 41.2 \\
Antenatal steroids (\%) & 83.6 & 76.4 \\
Postnatal steroids (\%) & 28.8 & 25.9 \\
Ventilator days (mean \pm SD) & $28.5 \pm 25.3$ & $24.3 \pm 25.0$ \\
Days on oxygen (mean \pm SD) & $57.4 \pm 39.8$ & $57.7 \pm 38.2$ \\
Sepsis (\%) & $52.9^{\mathrm{b}}$ & $41.6^{\mathrm{b}}$ \\
\hline Total number of controls with complete information varies by variable. ${ }^{\text {bStatistical }}$ \\
significant differences at 5\% level. & &
\end{tabular}

$\mathrm{ml}$ vs. 4,069 , range: $130-674,431 \mathrm{pg} / \mathrm{ml} ; P<0.05)$. These data are summarized in Table 2.

\section{Clinical Characteristics and Cytokines Associated With NEC}

In univariate logistic regression, the following variables were associated with NEC: gender, ethnicity, sepsis, birth weight, IL-2, IL-17, lymphotoxin- $\alpha$, MIP-1 $\alpha /$ CCL3, RANTES/CCL5, IL-8/CXCL8, TGF- $\beta_{1}$, and brain-derived neurotrophic factor. Each of these variables was included in a time-dependent multivariate survival model. After controlling for other covariates, gender, ethnicity, sepsis, IL-2, IL-8/CXCL8, and TGF- $\beta_{1}$ remained significant. A reduced model containing these 6 variables was fitted to the data (Table 3 ). In the case of ethnicity, African American was the reference cell and infants of non-Caucasian, non-African American ethnicity were at the highest risk (hazard ratio $=2.6 \times$ African-American infants) of NEC. Sepsis increased the risk of NEC by $46 \%$. Female gender and Caucasian race were protective, associated with a 23 and $35 \%$ lower risk, respectively. For every unit increase in TGF- $\beta_{1}$ and IL- 2 concentration, the risk of NEC decreased by $0.1 \%$.

\section{Blood Cytokines Before Onset of NEC}

To determine whether infants in the NEC group showed a unique cytokine signature even before they developed NEC, we compared samples from the NEC group drawn before onset of NEC vs. controls (Figure 1). The NEC group showed lower TGF- $\beta$ levels than controls at all time points (in controls, D1: median: 1,090, range: $50-7,305 \mathrm{pg} / \mathrm{ml}$ vs. 1,301 , range: $56-7,380$ pg/ml; D3: 988, range: $50-6,869$ vs. 1286 , range: $0-7,095$ pg/ml; D7: 1,073, range: $50-5,364$ vs. 1,487, range: 9-6,978 pg/ml; D14: 1,573, range: $50-5,677$ vs. 1,952 , range: $50-10,022 \mathrm{pg} / \mathrm{ml}$; D21: 1,701, range: $98-11,431$ vs. 2,129 , range: $50-12,750 \mathrm{pg} / \mathrm{ml} ; P<$ 0.05 for all subgroups). The NEC group also had lower IL-18, MIP-1 $\alpha / C C L 3$, RANTES/CCL5, and matrix metalloproteinase-9 on D3, lower MIP-1 $\alpha /$ CCL3 and RANTES/CCL5 on D7, and lower IL-17 levels on D21 (Table 4).

\section{Association of TGF- $\beta$ With NEC}

To determine whether blood TGF- $\beta$ levels could discriminate between the NEC and control groups, we computed receiver operating characteristics of TGF- $\beta$ using data from all the cases before they developed NEC and controls (Figure 2). The area under the curve was 0.67 , indicating "fair" diagnostic accuracy. Inclusion of other clinical variables or cytokines did not improve the accuracy of this model. A "cutoff" TGF- $\beta$ of $1,380 \mathrm{pg} / \mathrm{ml}$ (at the best sensitivity and specificity coordinates) classified infants into the NEC or control group with $64.0 \%$ accuracy (95\% confidence interval: 60.9-66.9\%; Table 5). The sensitivity and specificity were 68 and $46 \%$ on D1 and D3, 64 and 56\% on D7, and 40 and $74.4 \%$ on D14 and D21, respectively. The area under the curve improved to 0.71 when receiver operating characteristics were computed using cumulative TGF- $\beta$ values.

To confirm that low TGF- $\beta$ levels in infants who went on to develop NEC were not an artifact arising from exclusion of samples from later time points, we compared TGF- $\beta$ levels 


\section{Articles | Maheshwariet al.}

Table 2. Summary statistics for peak cytokine concentrations in cases and controls

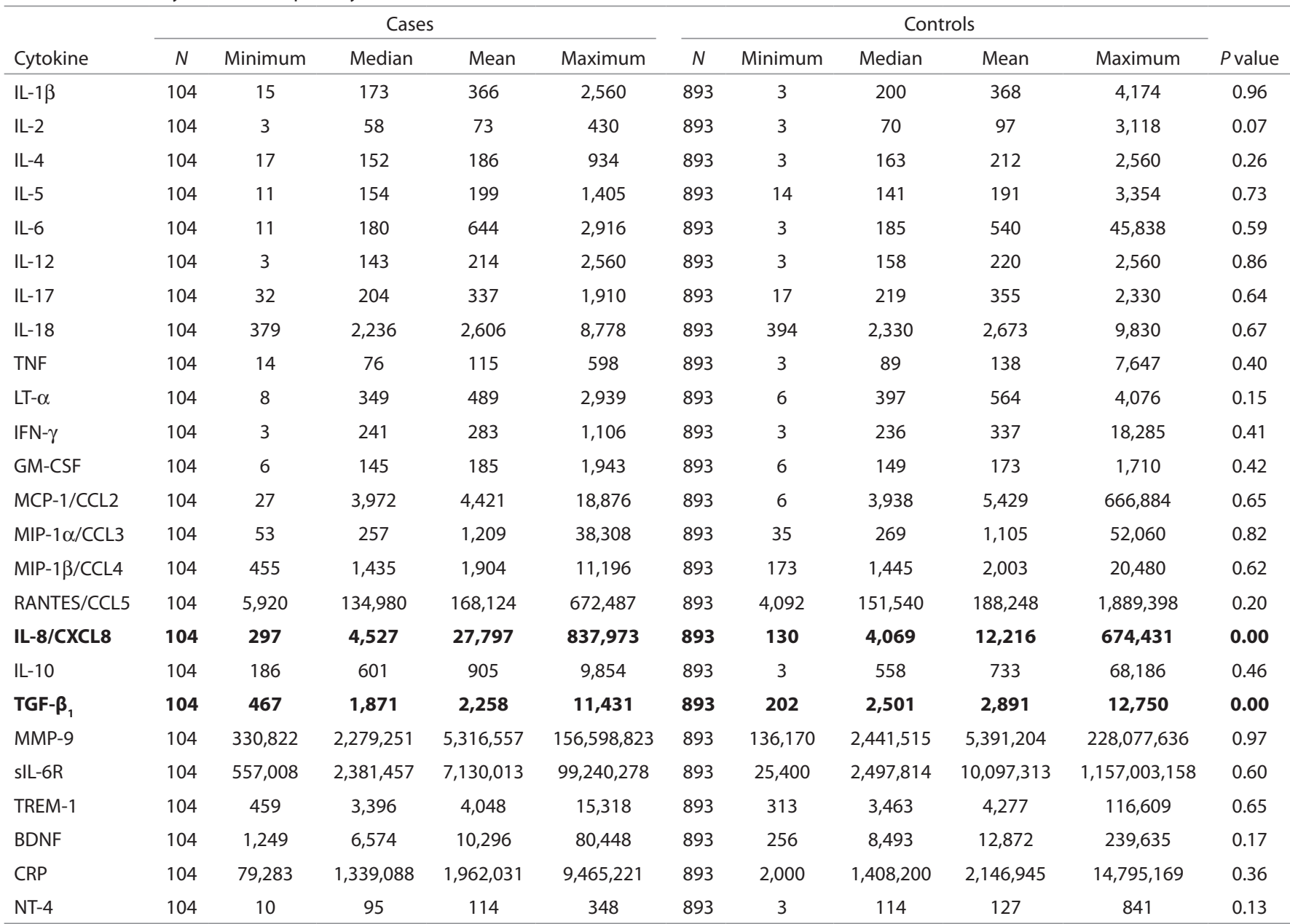

Peak was calculated as the maximum cytokine for each infant. For cases, peak was calculated using all sample days. For controls, peak was calculated using all sample days. Testing the average values, test is a two-sample $t$-test. Boldfaced numbers highlight statistically significant differences.

BDNF, brain-derived neurotrophic factor; CCL2, CC-motif ligand-2; CRP, C-reactive protein; CXCL8, CXC-motif ligand 8; GM-CSF, granulocyte macrophage colony-stimulating factor; IFN- $\gamma$, interferon- $\gamma$; IL, interleukin; LT-a, lymphotoxin-a; MCP-1, monocyte chemoattractant protein-1; MIP-1a, macrophage inflammatory protein-1a; MMP-9, matrix

metalloproteinase-9; NT4, neurotrophin-4; RANTES, regulated on activation, normal T-cell expressed and secreted; sIL-6R, soluble IL-6 receptor; TGF- $\beta_{1}$, transforming growth factor- $\beta_{1}$;

TNF, tumor necrosis factor; TREM-1, triggering receptor expressed on myeloid cells-1.

Table 3. Estimated multiplicative effect (SE) and test statistics for $\mathrm{HO}$ : $\beta=0$ for multivariate model

\begin{tabular}{lccc}
\hline Variable & Coefficient & $\begin{array}{c}\text { Exponentiated } \\
\text { coefficient }(S E)\end{array}$ & $\begin{array}{c}\text { Wald test } \\
(P \text { value })\end{array}$ \\
\hline Gender & -0.25452 & $0.77529(0.09689)$ & $-2.627(0.009)$ \\
Ethnicity (Caucasian) & -0.43201 & $0.64921(0.10164)$ & $-4.250(<0.001)$ \\
Ethnicity (other) & 0.95866 & $2.60820(0.24519)$ & $3.909(<0.001)$ \\
Sepsis & 0.38161 & $1.46464(0.09697)$ & $3.935(<0.001)$ \\
IL-2 & -0.00444 & $0.99557(0.00116)$ & $-3.815(<0.001)$ \\
IL-8/CXCL8 & 0.000003 & $1.00000(0.000001)$ & $3.065(0.002)$ \\
TGF- $\beta_{1}$ & -0.00019 & $0.99981(0.00005)$ & $-4.141(<0.001)$ \\
\hline
\end{tabular}

Log likelihood $=-2,806.555$.

CXCL8, CXC-motif ligand 8; IL, interleukin; TGF- $\beta_{1}$, transforming growth factor- $\beta_{1}$.

in the NEC vs. control groups as a function of postmenstrual age (PMA). NEC group had lower TGF- $\beta$ levels at PMA of $25 \mathrm{wk}$ (median: 897 , range: $50-7,305 \mathrm{pg} / \mathrm{ml}$ vs. 1,382 , range: $50-7,023 \mathrm{pg} / \mathrm{ml}$ in controls; $P=0.02$ ) and $27 \mathrm{wk}$ (median:
1,073 , range: $50-5,677 \mathrm{pg} / \mathrm{ml}$ vs. 1,646 , range: $9-9,484 \mathrm{pg} / \mathrm{ml}$; $P<0.0001)$ and showed a trend toward lower levels at PMA of 26 and $28-29 \mathrm{wk}$. The number of samples at other PMAs was not adequate for analysis.

TGF- $\beta$ levels showed a small but significant negative correlation with the postnatal age at first enteral feed (Pearson's $r=-0.09$ in complete data set; $P=0.007)$ and when full enteral feeds were achieved $(r=-0.11 ; P=0.001)$. TGF- $\beta$ levels also correlated negatively with death before discharge $(r=-0.14 ; P<0.0001)$.

\section{Changes in Cytokine Concentrations After the Onset of NEC}

To identify cytokine biomarkers of NEC, we compared samples drawn from the NEC group before vs. after the onset of NEC. Summary statistics for cytokines that showed significant differences on D7, D14, and D21 are depicted in Table 6. There was a trend toward lower TGF- $\beta$ levels in samples drawn after NEC, but the difference did not reach statistical significance. 


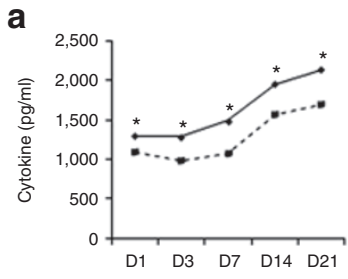

e

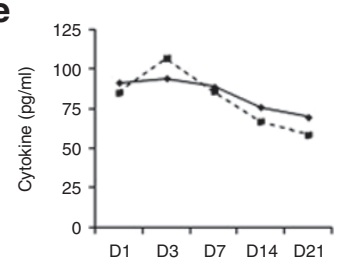

i

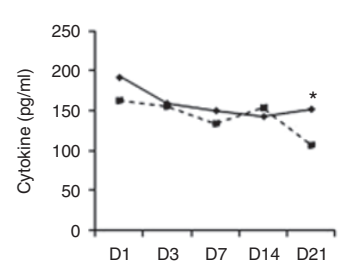

m

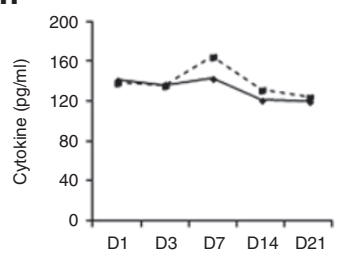

q

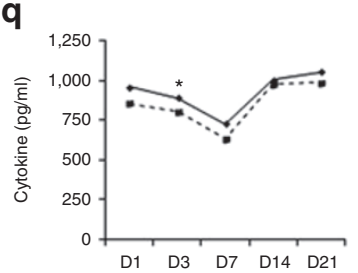

u

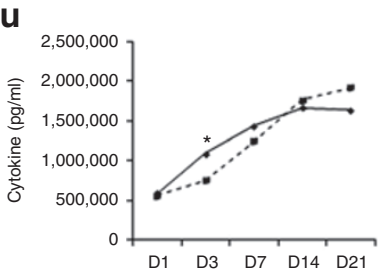

y

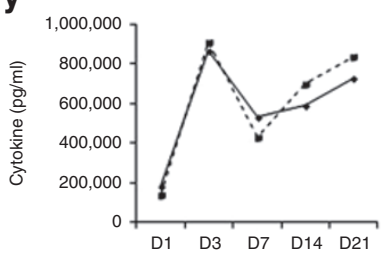

b

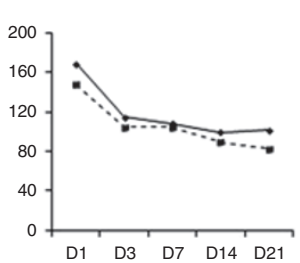

f

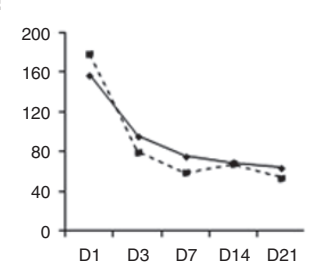

$\mathbf{j}_{2}$

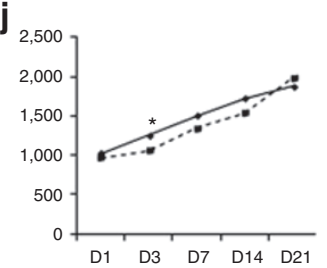

n

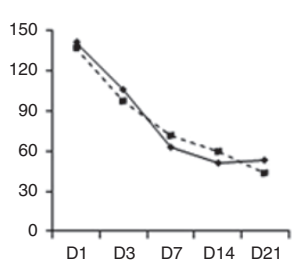

r

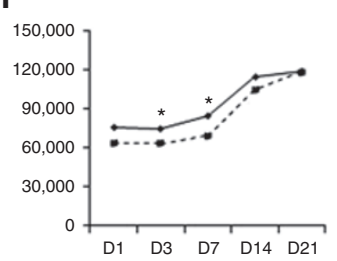

v

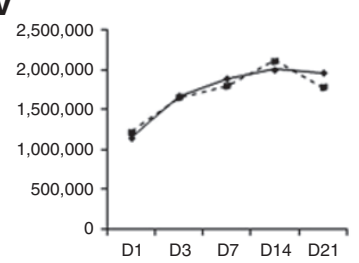

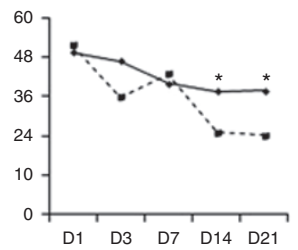

$\mathbf{g}_{500}$

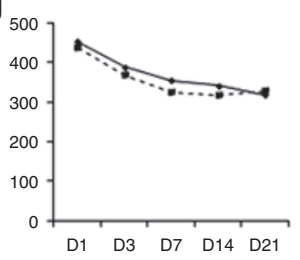

k

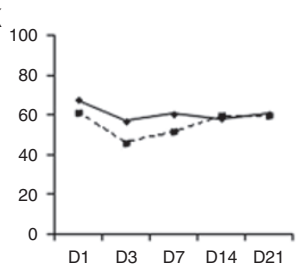

0

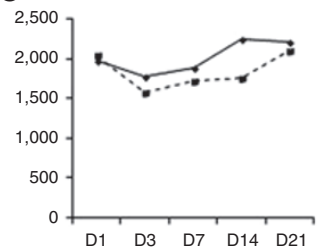

s

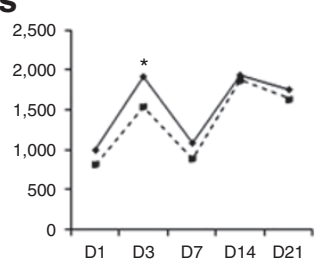

W

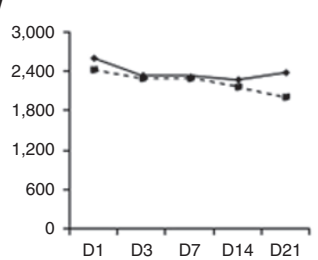

d

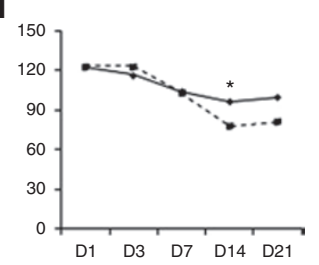

h

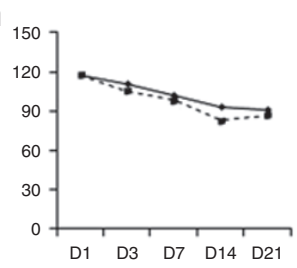

I

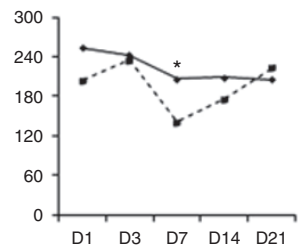

p

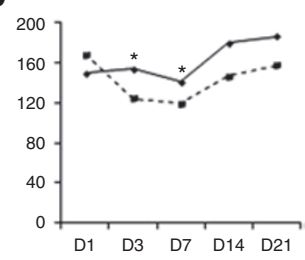

t

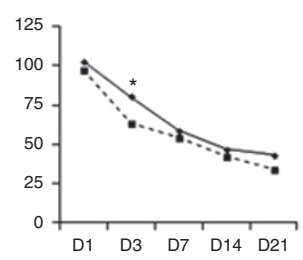

X

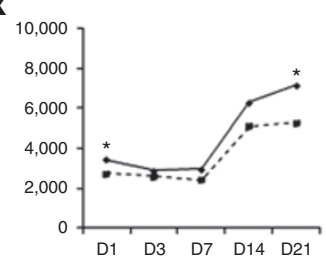

Figure 1. Differential expression of cytokines in blood spots from infants in the necrotizing enterocolitis (NEC; drawn before the onset of NEC; broken lines) and control groups (solid lines). Line diagrams depict median cytokine values on day 1 (D1), D3, D7, D14, and D21, not adjusted for other cytokines or clinical variables: (a) transforming growth factor- $\beta_{1}$, (b) interleukin (IL)-1 $\beta$, (c) IL-2, (d) IL-4, (e) IL-5, (f) IL-6, (g) IL-10, (h) IL-12, (i) IL-17, (j) IL-18, (k) tumor necrosis factor, (I) lymphotoxin- $\alpha,(\mathbf{m})$ interferon- $\gamma,(\mathbf{n})$ granulocyte macrophage colony-stimulating factor, (o) monocyte chemoattractant protein- $1 /$ CC-motif ligand-2 (CCL2), (p) macrophage inflammatory protein (MIP)-1 $\alpha / C C L 3,(\mathbf{q})$ MIP-1 $\beta / C C L 4$, (r) regulated on activation, normal T-cell expressed and secreted/CCL5, (s) IL-8/ CXC-motif ligand 8, (t) neurotrophin-4, (u) matrix metalloproteinase-9, (v) soluble IL-6 receptor, (w) triggering receptor expressed on myeloid cells-1, (x) brain-derived neurotrophic factor, (y) C-reactive protein. ${ }^{*} P<0.05$. 


\section{Articles | Maheshwariet al.}

Table 4. Summary statistics for cytokine concentrations in cases before NEC vs. controls summarized by day of measurement

\begin{tabular}{|c|c|c|c|c|c|c|c|c|c|c|c|}
\hline \multirow[b]{2}{*}{ Cytokine } & \multicolumn{5}{|c|}{ Cases before onset of NEC } & \multicolumn{5}{|c|}{ Controls } & \multirow[b]{2}{*}{$P$ value } \\
\hline & $N$ & Minimum & Median & Mean & Maximum & $N$ & Minimum & Median & Mean & Maximum & \\
\hline & \multicolumn{11}{|c|}{ Postnatal day 1} \\
\hline \multirow[t]{2}{*}{ TGF- $\beta$} & 61 & 50 & 1,090 & 1,272 & 7,305 & 470 & 56 & 1,301 & 1,586 & 7,380 & 0.02 \\
\hline & \multicolumn{11}{|c|}{ Postnatal day 3} \\
\hline MIP-1 $\alpha / C C L 3$ & 90 & 6 & 124 & 705 & 19,945 & 837 & 6 & 154 & 677 & 50,534 & 0.02 \\
\hline MMP-9 & 90 & 21,149 & 751,355 & $1,827,173$ & $37,067,238$ & 837 & 21,096 & $1,087,801$ & $2,294,486$ & $185,309,104$ & 0.01 \\
\hline RANTES/CCL5 & 90 & 2,159 & 63,127 & 74,585 & 417,270 & 837 & 1,590 & 75,081 & 94,666 & 678,668 & 0.03 \\
\hline \multirow[t]{2}{*}{ TGF- $\beta$} & 90 & 50 & 988 & 1,205 & 6,869 & 836 & 0 & 1,286 & 1,540 & 7,095 & 0.03 \\
\hline & \multicolumn{11}{|c|}{ Postnatal day 7} \\
\hline TGF- $\beta$ & \multicolumn{11}{|c|}{ Postnatal day 14} \\
\hline IL-2 & 68 & 3 & 25 & 36 & 155 & 808 & 3 & 37 & 54 & 616 & 0.01 \\
\hline IL-4 & 68 & 3 & 78 & 104 & 693 & 805 & 1 & 97 & 134 & 2,560 & 0.04 \\
\hline \multirow[t]{2}{*}{ TGF- $\beta$} & 68 & 50 & 1,573 & 1,855 & 5,677 & 808 & 50 & 1,952 & 2,259 & 10,022 & 0.04 \\
\hline & \multicolumn{11}{|c|}{ Postnatal day 21} \\
\hline IL-2 & 52 & 3 & 24 & 35 & 171 & 748 & 2 & 38 & 54 & 560 & 0.04 \\
\hline IL-17 & 52 & 3 & 107 & 163 & 737 & 748 & 1 & 153 & 229 & 2,330 & 0.04 \\
\hline BDNF & 52 & 425 & 5,309 & 9,808 & 80,448 & 749 & 24 & 7,176 & 10,468 & 239,635 & 0.05 \\
\hline
\end{tabular}

BDNF, brain-derived neurotrophic factor; CCL3, CC-motif ligand-3; IL, interleukin; MIP-1a, macrophage inflammatory protein-1 a; MMP-9, matrix metalloproteinase-9; NEC, necrotizing enterocolitis; RANTES, regulated on activation, normal T-cell expressed and secreted; TGF- $\beta$, transforming growth factor- $\beta$.

\section{DISCUSSION}

We identified male gender, non-Caucasian/non-African American ethnicity, history of sepsis, lower blood TGF- $\beta_{1}$ and IL-2 levels, and higher IL-8/CXCL8 levels to be associated with NEC in ELBW infants. Although infants who went on to develop NEC showed important differences in blood cytokine levels compared with controls starting on the day of birth, it was the clinical parameters, and not cytokines, that accounted for most of the risk of NEC in our statistical models. These findings are consistent with earlier observations in bronchopulmonary dysplasia (15), indicating that clinical variables may be driving the association of these cytokines with NEC, rather than vice versa. Our observations of increased risk of NEC in male infants and in certain ethnic groups are consistent with previous reports $(16,17)$. The risk of NEC in non-Caucasian/ non-African-American infants is consistent with the recent data showing higher NEC-related morbidity in Latinos (17), but not with earlier studies that identified African-American neonates to be at the highest risk $(16,17)$. Although some of these disparities could be due to differences in access to prenatal care, specific genetic factors may also be at play.

In our study, ELBW infants who went on to develop NEC started with low circulating TGF- $\beta_{1}$ since D1. Blood TGF- $\beta_{1}$
Table 5. Diagnostic value of TGF- $\beta_{1}<1,380 \mathrm{pg} / \mathrm{ml}$ as a predictive marker for NEC

\begin{tabular}{llcc}
\hline & & \multicolumn{2}{c}{$95 \%$ Confidence interval } \\
\cline { 3 - 4 } & & Lower limit & Upper limit \\
\hline Prevalence & 0.1 & 0.09 & 0.12 \\
Sensitivity & 0.61 & 0.51 & 0.7 \\
Specificity & 0.64 & 0.61 & 0.67
\end{tabular}

For any particular test result, the probability that it will be:

$\begin{array}{llll}\text { Positive } & 0.38 & 0.35 & 0.41 \\ \text { Negative } & 0.62 & 0.59 & 0.65\end{array}$

For any particular positive test result, the probability that it is:

$\begin{array}{llll}\text { True positive } & 0.16 & 0.13 & 0.21 \\ \text { False positive } & 0.84 & 0.79 & 0.87\end{array}$

For any particular negative test result, the probability that it is:

$\begin{array}{llll}\text { True negative } & 0.93 & 0.91 & 0.95 \\ \text { False negative } & 0.07 & 0.05 & 0.09\end{array}$

Likelihood ratios

$\begin{array}{llll}\text { Positive } & 1.71 & 1.43 & 2.04\end{array}$

$\begin{array}{llll}\text { Negative } & 0.6 & 0.47 & 0.77\end{array}$

NEC, necrotizing enterocolitis; TGF- $\beta$, transforming growth factor- $\beta$. 


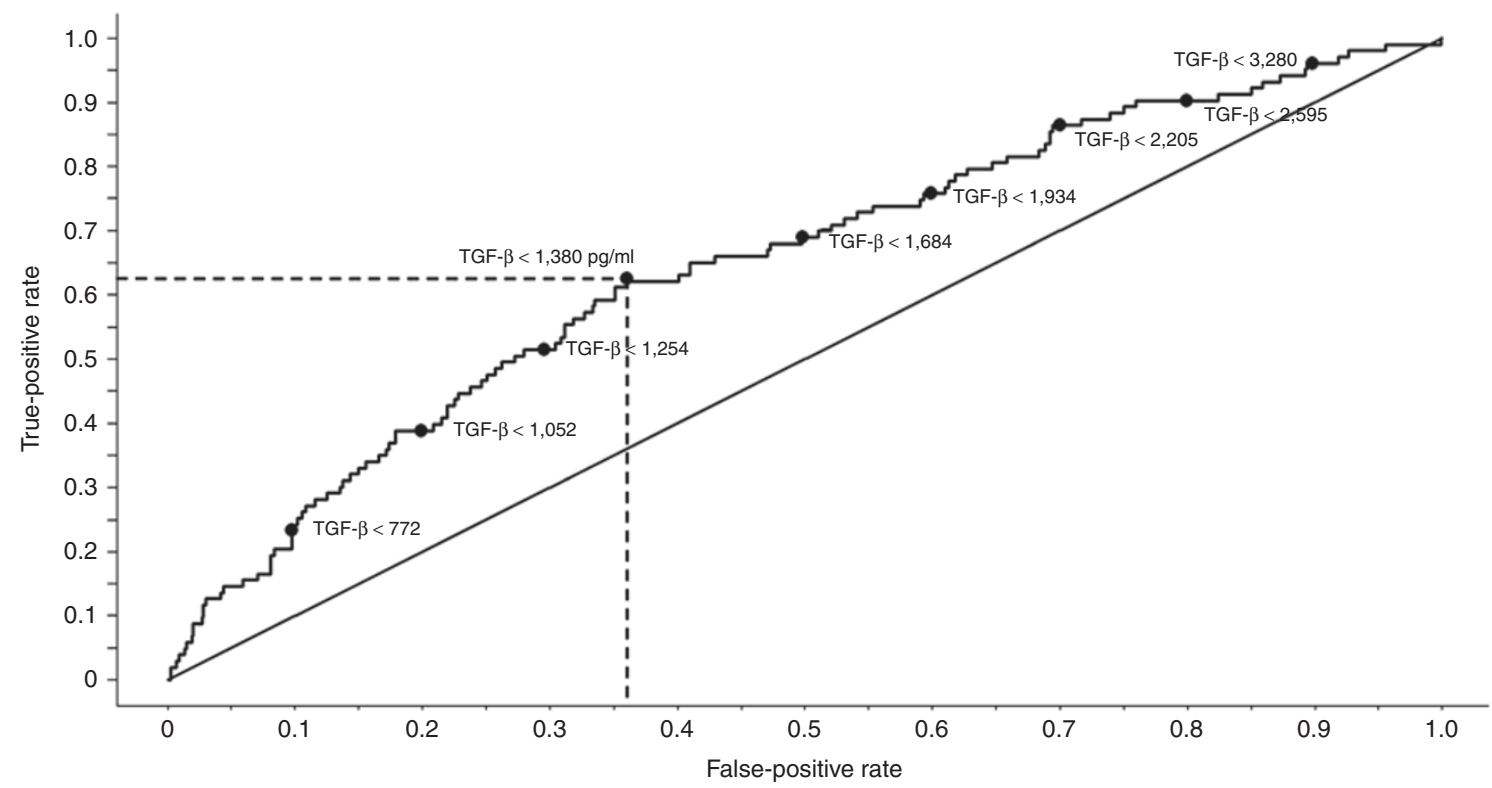

Figure 2. Receiver operating characteristics of transforming growth factor (TGF)- $\beta$ in blood spots from infants in the necrotizing enterocolitis (NEC; drawn before the onset of NEC) and control groups. Black circles on the curve indicate TGF- $\beta$ concentrations. A cutoff TGF- $\beta$ level of $1,380 \mathrm{pg} / \mathrm{ml}$ (marked by broken lines in the figure) correctly classified $61.2 \%$ infants in the NEC group (true positives) but gave a false-positive result in $35.7 \%$ controls.

Table 6. Cytokine concentrations in the NEC group before and after onset of NEC

\begin{tabular}{|c|c|c|c|c|c|c|c|c|c|c|c|}
\hline \multirow[b]{2}{*}{ Cytokine } & \multicolumn{5}{|c|}{ Samples drawn from infants who were yet to develop NEC } & \multicolumn{5}{|c|}{ Samples drawn from infants who had already developed NEC } & \multirow[b]{2}{*}{$P$ value } \\
\hline & $N$ & Minimum & Median & Mean & Maximum & $N$ & Minimum & Median & Mean & Maximum & \\
\hline & \multicolumn{11}{|c|}{ Postnatal day 7} \\
\hline & & & & & & tnata & ay 14 & & & & \\
\hline IL-1 $\beta$ & 68 & 3 & 89 & 176 & 860 & 23 & 3 & 132 & 334 & 2,560 & 0.05 \\
\hline MCP-1/CCL2 & 68 & 21 & 1,761 & 2,698 & 15,639 & 23 & 242 & 3,668 & 4,478 & 18,876 & 0.02 \\
\hline MIP-1 $\beta / C C L 4$ & 68 & 6 & 978 & 1,242 & 5,749 & 23 & 460 & 1,165 & 2,137 & 11,196 & 0.02 \\
\hline IL-8/CXCL8 & 68 & 206 & 1,872 & 5,183 & 102,627 & 23 & 786 & 8,233 & 26,107 & 280,505 & 0.01 \\
\hline \multirow[t]{2}{*}{ IL-10 } & 68 & 3 & 316 & 353 & 997 & 23 & 33 & 397 & 844 & 5,022 & 0.00 \\
\hline & \multicolumn{11}{|c|}{ Postnatal day 21} \\
\hline
\end{tabular}

CCL2, CC-motif ligand-2; CRP, C-reactive protein; CXCL8, CXC-motif ligand 8; IL, interleukin; MCP-1, monocyte chemoattractant protein-1; MIP-1 a, macrophage inflammatory protein-1a; NEC, necrotizing enterocolitis.

concentrations $<1,380 \mathrm{pg} / \mathrm{ml}$ predicted NEC with $64 \%$ accuracy. Although several biomarkers (such as the inter- $\alpha$ inhibitor protein, intestinal fatty acid-binding protein, hexosaminidase, proapolipoprotein CII, and des-arginine serum amyloid A) have been identified for their ability to discriminate between confirmed NEC and other causes of feeding intolerance (18-21), blood TGF- $\beta_{1}$ is the first biomarker to estimate the risk of NEC in a newly born premature infant. Despite moderate diagnostic accuracy, the ability of blood TGF- $\beta_{1}$ to identify at-risk infants several weeks before the actual development of NEC has the potential to change clinical practice, allowing targeted application of splanchnic perfusion/oxygenation monitors, devices that are potentially useful but are expensive or cumbersome for universal use in neonatal intensive care units, or the use of banked human milk and probiotics, interventions that are promising but of unproven safety in ELBW infants $(22,23)$. Although a predictive accuracy of $64 \%$ may seem modest, it may be a major advancement in our ability to estimate the risk of NEC in a premature infant on the first postnatal day-a rise from our current accuracy levels of $5-15 \%$ based on data on incidence of NEC by gestational age. The negative predictive value of $93 \%$ is similarly modest (in a 


\section{Articles | Maheshwarietal.}

cohort with $10 \%$ incidence of NEC, a randomly chosen sample should show an negative predictive value of $90 \%$ ) but may be clinically important; most premature infants with abdominal signs are currently treated presumptively for NEC even when they do not have NEC, and in this context, even a small improvement in risk stratification may be useful.

Our findings of low blood TGF- $\beta$ levels in the NEC group are consistent with our previous reports in which we showed that preterm neonates may be at risk of inflammatory mucosal injury and NEC because of a developmental deficiency of TGF$\beta$ isoforms TGF- $\beta_{1}$ and TGF- $\beta_{2}$ in the intestine $(12,24)$. We demonstrated that TGF- $\beta$ normally suppresses the inflammatory responses of resident intestinal macrophages, thereby promoting mucosal tolerance to bacterial products (12). However, in the TGF- $\beta$-deficient preterm intestine, macrophages produce an exaggerated cytokine response upon exposure to bacterial products (12). In addition to its effects on macrophages, TGF- $\beta$ also restricts the Th 1 and Th 2 gut lymphocyte pools by suppressing T-cell trafficking, increasing apoptosis in activated $\mathrm{T}$ cells, and promoting the development of regulatory $\mathrm{T}$ cells (25). The anti-inflammatory effects of TGF- $\beta$ are evident in TGF- $\beta_{1}$-deficient mice, which develop mucosal and systemic inflammation within a few weeks after birth (26).

Circulating TGF- $\beta$ originates from diverse cellular sources including platelets, monocytes/macrophages, lymphocytes, epithelium, and mesenchymal cells (25) and comprised mostly latent TGF- $\beta$ bound to $\alpha 2$-macroglobulin along with a small pool of free, active TGF- $\beta$ that is rapidly taken up and degraded in peripheral tissues. In infants who developed NEC, there are 3 possible explanations for the low circulating TGF- $\beta$ levels. The first is increased peripheral uptake of TGF- $\beta$ to compensate for low tissue expression. Peripheral consumption of TGF- $\beta$ has been described in patients with Guillain-Barré syndrome who have low plasma TGF- $\beta$ levels despite evidence of increased production (27). Consistent with this hypothesis, we have previously shown that TGF- $\beta$ expression is decreased in healthy margins of tissues resected for NEC (12). A second explanation for low circulating TGF- $\beta$ levels is based on an assumption that the developing intestine is a major contributor to circulating TGF- $\beta$ levels. Because TGF- $\beta$ expression increases in the intestine as a function of gestational maturation, lower tissue expression of TGF- $\beta$ in infants who developed NEC could conceivably reflect an underlying state of arrested mucosal development, whereas low TGF- $\beta$ expression may indicate persistence of a cytokine profile corresponding to an earlier, less mature developmental epoch $(28,29)$. In support of this possibility, we detected lower TGF- $\beta$ levels in NEC group than in controls at the same PMA. Finally, infants who developed NEC may constitutively produce less TGF- $\beta$ than controls due to genetic/epigenetic factors. Polymorphisms in the TGF- $\beta 1$ gene such as G915C, C-509T, and T869C are common in the general population and are associated with higher blood TGF$\beta$ levels (30), although the significance of these genetic markers is unclear in NEC. We have recently shown that NEC is associated with dimethylation of the lysine 9 residue of histone 3 in the TGF- $\beta$ nucleosome, a repressive modification associated with facultative heterochromatin assembly and transcriptional silencing (24). Further study is needed to determine the timing of these epigenetic changes in at-risk infants.

We did not find support for our second hypothesis as we did not detect elevated cytokine levels at early time points in the NEC group. Before the development of NEC, infants in the NEC group had significantly low IL-18, MIP-1//CCL4, and RANTES/CCL5 and a tendency for most other cytokines to be lower than controls. Interestingly, NEC is marked by a paucity of $\mathrm{T}$ cells, which are a major cellular source of many of these cytokines (31-33). We also detected low IL-2 levels in our patients with NEC on D14 and D21. IL-2 is a Th1-derived cytokine that promotes proliferation and activation of $\mathrm{CD} 4^{+}$ and $\mathrm{CD} 8^{+}$lymphocytes, and an IL-2-deficient state may result in limited T-cell lifespan and proliferation. However, low levels of IL- 6 and CRP, which are mainly produced in the liver, indicate that these defects may extend beyond the intestine (34).

The diagnosis of NEC was associated with increased expression of IL-6, IL-8/CXCL8, IL-10, IL-18, monocyte chemoattractant protein-1/CCL2, MIP-1ß/CCL3, CRP, and NT-4. Elevated IL-8/CXCL8, IL-6, and IL-18 levels have been noted previously during NEC $(3-6,35)$ and are adversely associated with disease severity and with short-term survival in NEC $(4,36)$. IL-6 has a shorter half-life and may be detectable only for short periods after onset of NEC (37), whereas IL-8/CXCL8 levels may show sustained elevation and correlate with the severity of illness over a period of time (5). Increased IL- 6 and CRP in NEC support the presence of a gut-liver inflammatory axis, where the transfer of bacterial products and inflammatory mediators into the portal circulation amplifies the production of cytokines and nitric oxide in the liver. These mediators then exacerbate intestinal injury via enterohepatic circulation, setting up a feedforward loop of inflammation (38).

Major strengths of our study are a large sample of ELBW infants recruited from multiple sites, prospective data collection by trained observers, and measurement of multiple cytokines at serial time points, including well before the onset of NEC. An important limitation is its exclusive reliance on circulating levels of cytokines, which does not identify the cellular sources or the effector cells that are likely to respond to these cytokines. We were also limited by exclusion of infants with early-onset sepsis $(n=16)$ who would have comprised an important comparison group. We were unable to evaluate the effects of human milk vs. formula feeding, gut microbiota, and chorioamnionitis, each of which may alter or reprogram infant cytokine profiles, due to limitations of the retrospective study design. Finally, the National Institute of Child Health and Human Development Cytokine Study was designed to investigate cytokine predictors of neurodevelopmental impairment, and therefore, the cytokine panel comprised analytes generally associated with systemic inflammation and not specifically with gut mucosal injury. This panel included TGF- $\beta_{1}$ but not TGF- $\beta_{2}$, and further study is needed to determine the relationship of blood TGF- $\beta_{2}$ levels with NEC. It may also be possible to improve the predictive accuracy of blood TGF- $\beta$ levels by combining these data with other clinical predictors of NEC 
(2) such as absence/reversal of umbilical blood flow on prenatal Doppler examination or histopathological evidence of chorioamnionitis.

\section{Conclusions}

Male gender, non-Caucasian/non-African American ethnicity, sepsis, lower blood TGF- $\beta_{1}$ and IL-2 levels, and higher IL-8 levels were associated with NEC. Blood TGF- $\beta$ concentrations $<1,380 \mathrm{pg} / \mathrm{ml}$ predicted NEC with about $64 \%$ accuracy on the day of birth. We did not find support for our second hypothesis that infants who develop NEC have higher cytokine levels in early neonatal period.

\section{METHODS}

We conducted a secondary analysis of clinical and biological data collected as part of the National Institute of Child Health and Human Development Cytokine Study (11). The study was approved by the Institutional Review Board at the University of Alabama at Birmingham and at all other participating institutions. Preterm neonates with birth weight of 401-1,000 g were enrolled after obtaining written informed consent from the parent(s). Whole blood spots were collected on standardized filter paper and frozen on postnatal days $0-1$ (D1), $3 \pm 1$ (D3), $7 \pm 1$ (D7), $14 \pm 3$ (D14), and $21 \pm 3$ (D21) using an established protocol that has been shown to maintain sample quality and consistency for cytokine measurements over extended periods of time ( $>20$ y) (39). Clinical data were collected by trained research coordinators and analyzed at a central data coordinating center. Concentrations of 25 cytokines/inflammatory mediators were measured in blood spot eluates using a multiplex flow cytometric immunoassay based on laser detection of color-encoded antibody-tagged microspheres (xMAP assay, Luminex, Austin, TX) (39). The following analytes were included in the immunoassay panel: IL-1 $\beta$, IL-2, IL-4, IL-5, IL-6, IL-12, IL-17, IL-18, tumor necrosis factor, lymphotoxin$\alpha /$ tumor necrosis factor- $\beta$, interferon- $\gamma$, granulocyte macrophage colony-stimulating factor, monocyte chemoattractant protein-1/CCL2, MIP-1 $\alpha / C C L 3$, MIP-1 $\beta / C C L 4$, RANTES/CCL5, IL-8/CXCL8, IL-10, TGF- $\beta_{1}$, matrix metalloproteinase- 9 , soluble IL- 6 receptor, triggering receptor expressed on myeloid cells-1, brain-derived neurotrophic factor, neurotrophin-4, and CRP. This assay has low intra- $(<10 \%)$ and interassay $(7-23 \%)$ variation and has lower limits of detection lower than reported median plasma concentrations of these cytokines/ inflammatory factors in normal neonates.

The "NEC group" comprised all preterm neonates enrolled in the National Institute of Child Health and Human Development Cytokine Study with a diagnosis of NEC (Bell's stages II and III) (40). All other infants were included in the control group. Demographic and clinical data, including gestational age, birth weight, gender, ethnicity, history of sepsis, postnatal age when feeds were started, age when full enteral feeds were achieved, and age at onset of NEC, were obtained from the Neonatal Research Network generic database. Frequencies (percentage), means, SDs, median values, and ranges were computed for demographic data and cytokine concentrations. Frequencies were compared by the Fisher's exact test. We compared (i) peak cytokine concentrations in NEC vs. control groups, (ii) cytokine concentrations in NEC group before onset of NEC vs. controls, and (c) samples drawn from the NEC group "before NEC" vs. "after NEC" group, using the Student's $t$-test or when the variance in the two groups was unequal, the Welch-Satterthwaite $t$-test.

To identify clinical characteristics and cytokines associated with NEC, we performed a stepwise logistic regression analysis. Univariate logistic regression models were first used to evaluate the association between NEC and categorical predictors such as gender $(1=$ male, 2 = female), ethnicity ( 1 = African American, $2=$ Caucasian, $3=$ other), and sepsis $(1=$ yes, $0=$ no), and then with continuous predictors (gestational age, birth weight, and cytokines). Variables identified in the univariate analysis at $P<0.20$ (and some other covariates that were not significant but were biologically plausible, such as gender) were then used to develop a multivariate survival model. Cytokines were considered as time-dependent and right-censored (because many infants developed NEC after the end of the study period) covariates. For categorical predictors, we examined Kaplan-Meier curves and then used the log-rank test for equality across strata to identify predictors $(P<0.25)$ to be included in the final model. For continuous variables, a univariate Cox proportional hazard regression model was used to identify predictors to be included in the multivariate analysis.

To determine the diagnostic usefulness of blood TGF- $\beta$ levels, we computed receiver operating characteristics and selected a TGF- $\beta$ concentration with the highest sum of sensitivity and specificity for further evaluation as a diagnostic "cutoff" value. The accuracy of this cutoff value in correctly classifying infants into the NEC and control groups was computed using a $2 \times 2$ confusion matrix of the predicted and observed number of cases. To determine whether the diagnostic accuracy of TGF- $\beta$ could be improved by adding other cytokines or clinical characteristics to the receiver operating characteristic model, we performed logistic regression and identified covariates associated with NEC at $P<0.20$. These covariates were then evaluated using inclusive (with all variables) and reduced models.

\section{ACKNOWLEDGMENTS}

A.M., R.L.S., R.A.D., and W.A.C. conceptualized and designed the study and wrote the manuscript. B.M.-H., A.D., and S.A.M. maintained the database and performed statistical analysis. P.T., K.S., and D.M.H. provided critical expertise on methodological issues. R.D.H. provided critical expertise in study design and execution. All the authors reviewed and approved the manuscript. The following investigators participated in this study: Alan Jobe, University of Cincinnati; William Oh, Abbot R. Laptook, Lewis P. Rubin, Angelita M. Hensman, Brown University; Avroy A. Fanaroff, Michele C. Walsh, Nancy S. Newman, Bonnie S. Siner, Case Western Reserve University; Diana E. Schendel, Centers for Disease Control and Prevention; Edward F. Donovan, Vivek Narendran, Barbara Alexander, Cathy Grisby, Marcia Worley Mersmann, Holly L. Mincey, Jody Hessling, Cincinnati Children's Hospital Medical Center; Ronald N. Goldberg, C. Michael Cotten, Kathy J. Auten, Duke University; Barbara J. Stoll, Ira Adams-Chapman, Ellen C. Hale, Emory University; Linda L. Wright, Rosemary D. Higgins, Sumner J. Yaffe, Elizabeth M. McClure, Eunice Kennedy Shriver National Institute of Child Health and Human Development; James A. Lemons, Brenda B. Poindexter, Diana D. Appel, Dianne E. Herron, Leslie D. Wilson, Indiana University; W. Kenneth Poole, Abhik Das, Scott A. McDonald, Betty Hastings, Kristin Zaterka-Baxter, Jeanette O'Donnell Auman, RTI International; David K. Stevenson, Krisa P. Van Meurs, M. Bethany Ball, Stanford University; Kristin Skogstrand, David M. Hougaard, Statens Serum Institut; Poul Thorsen, University of Aarhus, Denmark; Namasivayam Ambalavanan, Monica V. Collins, Shirley S. Cosby, University of Alabama at Birmingham; Neil N. Finer, Maynard R. Rasmussen, David Kaegi, Kathy Arnell, Clarence Demetrio, Wade Rich, University of California, San Diego; Charles R. Bauer, Shahnaz Duara, Ruth Everett-Thomas, University of Miami; Lu-Ann Papile, Conra Backstrom Lacy, University of New Mexico; Sheldon B. Korones, Henrietta S. Bada, Tina Hudson, University of Tennessee; Abbot R. Laptook, Walid A. Salhab, R. Sue Broyles, Susie Madison, Jackie F. Hickman, Sally S. Adams, Linda A. Madden, Elizabeth Heyne, Cristin Dooley, University of Texas Southwestern Medical Center at Dallas; Jon E. Tyson, Kathleen Kennedy, Brenda H. Morris, Esther G. Akpa, Patty A. Cluff, Claudia Y. Franco, Anna E. Lis, Georgia E. McDavid, Patti L. Tate, University of Texas Health Science Center at Houston; T. Michael O'Shea, Robert G. Dillard, Lisa K. Washburn, Barbara G. Jackson, Nancy J. Peters, Wake Forest University; G. Ganesh Konduri, Geraldine Muran, Rebecca Bara, Wayne State University; Richard A. Ehrenkranz, Patricia Gettner, Monica Konstantino, Elaine Romano, Yale University. The findings and conclusions in this report are those of the authors and do not necessarily represent the official position of the Centers for Disease Control and Prevention (CDC), Atlanta, GA.

\section{STATEMENT OF FINANCIAL SUPPORT}

The National Institutes of Health (NIH), Bethesda, MD (General Clinical Research Center grants M01 RR30, M01 RR32, M01 RR39, M01 RR70, M01 RR80, M01 RR633, M01 RR750, M01 RR997, M01 RR6022, M01 RR7122, M01 RR8084, M01 RR16587), Eunice Kennedy Shriver National Institute of Child Health and Human Development, NIH (grants U01 HD36790, U10 HD21364, U10 HD21373, U10 HD21385, U10 HD21397, U10 HD21415, U10 HD27851, U10 HD27853, U10 HD27856, U10 HD27871, U10 HD27880, U10 HD27881, 
U10 HD27904, U10 HD34216, U10 HD40461, U10 HD40492, U10 HD40498, U10 HD40689), and the US Centers for Disease Control and Prevention, Atlanta, GA (interagency agreement Y1-HD-5000-01) provided grant support for recruitment during 1999-2001 and data analysis for the Neonatal Research Network's Cytokine Study. The funding agencies provided overall oversight for study conduct, but all data analysis and interpretation were independent of the funding agencies. The study was made possible in part by the NIH grant R01HD059142 (awarded to A.M.).

Disclosure: The authors have no financial relationships relevant to this article or other conflicts of interest to disclose.

\section{REFERENCES}

1. Stoll BJ, Hansen NI, Bell EF, et al.; Eunice Kennedy Shriver National Institute of Child Health and Human Development Neonatal Research Network. Neonatal outcomes of extremely preterm infants from the NICHD Neonatal Research Network. Pediatrics 2010;126:443-56.

2. Neu J, Walker WA. Necrotizing enterocolitis. N Engl J Med 2011;364: 255-64.

3. Viscardi RM, Lyon NH, Sun CC, Hebel JR, Hasday JD. Inflammatory cytokine mRNAs in surgical specimens of necrotizing enterocolitis and normal newborn intestine. Pediatr Pathol Lab Med 1997;17:547-59.

4. Harris MC, Costarino AT Jr, Sullivan JS, et al. Cytokine elevations in critically ill infants with sepsis and necrotizing enterocolitis. J Pediatr 1994;124:105-11.

5. Edelson MB, Bagwell CE, Rozycki HJ. Circulating pro- and counterinflammatory cytokine levels and severity in necrotizing enterocolitis. Pediatrics 1999;103:766-71.

6. Ng PC, Li K, Wong RP, et al. Proinflammatory and anti-inflammatory cytokine responses in preterm infants with systemic infections. Arch Dis Child Fetal Neonatal Ed 2003;88:F209-13.

7. Benkoe T, Baumann S, Weninger M, et al. Comprehensive evaluation of 11 cytokines in premature infants with surgical necrotizing enterocolitis. PLoS One 2013;8:e58720.

8. Hsueh W, Caplan MS, Tan X, MacKendrick W, Gonzalez-Crussi F. Necrotizing enterocolitis of the newborn: pathogenetic concepts in perspective. Pediatr Dev Pathol 1998;1:2-16.

9. Maheshwari A. Role of cytokines in human intestinal villous development. Clin Perinatol 2004;31:143-55.

10. Schelonka RL, Maheshwari A, Carlo WA, et al.; NICHD Neonatal Research Network. T cell cytokines and the risk of blood stream infection in extremely low birth weight infants. Cytokine 2011;53:249-55.

11. Carlo WA, McDonald SA, Tyson JE, et al.; Eunice Kennedy Shriver National Institute of Child Health and Human Development Neonatal Research Network. Cytokines and neurodevelopmental outcomes in extremely low birth weight infants. J Pediatr 2011;159:919-25.e3.

12. Maheshwari A, Kelly DR, Nicola T, et al. TGF- $\beta 2$ suppresses macrophage cytokine production and mucosal inflammatory responses in the developing intestine. Gastroenterology 2011;140:242-53.

13. Been JV, Rours IG, Kornelisse RF, et al. Histologic chorioamnionitis, fetal involvement, and antenatal steroids: effects on neonatal outcome in preterm infants. Am J Obstet Gynecol 2009;201:587.e1-8.

14. Andrews WW, Goldenberg RL, Faye-Petersen O, Cliver S, Goepfert AR, Hauth JC. The Alabama Preterm Birth study: polymorphonuclear and mononuclear cell placental infiltrations, other markers of inflammation, and outcomes in 23- to 32-week preterm newborn infants. Am J Obstet Gynecol 2006;195:803-8.

15. Ambalavanan N, Carlo WA, D’Angio CT, et al.; Eunice Kennedy Shriver National Institute of Child Health and Human Development Neonatal Research Network. Cytokines associated with bronchopulmonary dysplasia or death in extremely low birth weight infants. Pediatrics 2009;123:1132-41.

16. Holman RC, Stoll BJ, Clarke MJ, Glass RI. The epidemiology of necrotizing enterocolitis infant mortality in the United States. Am J Public Health 1997;87:2026-31.

17. Guner YS, Friedlich P, Wee CP, Dorey F, Camerini V, Upperman JS. State-based analysis of necrotizing enterocolitis outcomes. J Surg Res 2009;157:21-9.
18. Chaaban H, Shin M, Sirya E, Lim YP, Caplan M, Padbury JF. Inter-alpha inhibitor protein level in neonates predicts necrotizing enterocolitis. J Pediatr 2010;157:757-61.

19. Ng PC, Ang IL, Chiu RW, et al. Host-response biomarkers for diagnosis of late-onset septicemia and necrotizing enterocolitis in preterm infants. J Clin Invest 2010;120:2989-3000.

20. Edelson MB, Sonnino RE, Bagwell CE, Lieberman JM, Marks WH, Rozycki HJ. Plasma intestinal fatty acid binding protein in neonates with necrotizing enterocolitis: a pilot study. J Pediatr Surg 1999;34:1453-7.

21. Lobe TE, Richardson CJ, Rassin DK, Mills R, Schwartz M. Hexosaminidase: a biochemical marker for necrotizing enterocolitis in the preterm infant. Am J Surg 1984;147:49-52.

22. Alfaleh K, Anabrees J, Bassler D, Al-Kharfi T. Probiotics for prevention of necrotizing enterocolitis in preterm infants. Cochrane Database Syst Rev 2011;3:CD005496.

23. Quigley MA, Henderson G, Anthony MY, McGuire W. Formula milk versus donor breast milk for feeding preterm or low birth weight infants. Cochrane Database Syst Rev 2007;4:CD002971.

24. Namachivayam K, Blanco CL, MohanKumar K, et al. Smad7 inhibits autocrine expression of TGF- $\beta 2$ in intestinal epithelial cells in baboon necrotizing enterocolitis. Am J Physiol Gastrointest Liver Physiol 2013;304: G167-80.

25. Wahl SM. Transforming growth factor-beta: innately bipolar. Curr Opin Immunol 2007;19:55-62.

26. Yoshinaga K, Obata H, Jurukovski V, et al. Perturbation of transforming growth factor (TGF)-betal association with latent TGF-beta binding protein yields inflammation and tumors. Proc Natl Acad Sci USA 2008;105:18758-63.

27. Dahle C, Kvarnstrom M, Ekerfelt C, Samuelsson M, Ernerudh J. Elevated number of cells secreting transforming growth factor beta in GuillainBarré syndrome. APMIS 2003;111:1095-104.

28. Maheshwari A, Lacson A, Lu W, et al. Interleukin-8/CXCL8 forms an autocrine loop in fetal intestinal mucosa. Pediatr Res 2004;56:240-9.

29. Nanthakumar NN, Fusunyan RD, Sanderson I, Walker WA. Inflammation in the developing human intestine: A possible pathophysiologic contribution to necrotizing enterocolitis. Proc Natl Acad Sci USA 2000;97:6043-8.

30. Mak JC, Leung HC, Sham AS, et al. Genetic polymorphisms and plasma levels of transforming growth factor-beta(1) in Chinese patients with tuberculosis in Hong Kong. Cytokine 2007;40:177-82.

31. Anttila A, Kauppinen H, Koivusalo A, Heikkila P, Savilahti E, Rintala R. T-cell-mediated mucosal immunity is attenuated in experimental necrotizing enterocolitis. Pediatr Surg Int 2003;19:326-30.

32. Pender SL, Braegger C, Gunther U, et al. Matrix metalloproteinases in necrotising enterocolitis. Pediatr Res 2003;54:160-4.

33. MohanKumar K, Kaza N, Jagadeeswaran R, et al. Gut mucosal injury in neonates is marked by macrophage infiltration in contrast to pleomorphic infiltrates in adult: evidence from an animal model. Am J Physiol Gastrointest Liver Physiol 2012;303:G93-102.

34. Hsueh W, Caplan MS, Qu XW, Tan XD, De Plaen IG, Gonzalez-Crussi F. Neonatal necrotizing enterocolitis: clinical considerations and pathogenetic concepts. Pediatr Dev Pathol 2003;6:6-23.

35. Ford HR, Sorrells DL, Knisely AS. Inflammatory cytokines, nitric oxide, and necrotizing enterocolitis. Semin Pediatr Surg 1996;5:155-9.

36. Sharma R, Tepas JJ 3rd, Hudak ML, et al. Neonatal gut barrier and multiple organ failure: role of endotoxin and proinflammatory cytokines in sepsis and necrotizing enterocolitis. J Pediatr Surg 2007;42:454-61.

37. Lodha A, Asztalos E, Moore AM. Cytokine levels in neonatal necrotizing enterocolitis and long-term growth and neurodevelopment. Acta Paediatr 2010;99:338-43.

38. Halpern MD, Clark JA, Saunders TA, et al. Reduction of experimental necrotizing enterocolitis with anti-TNF-alpha. Am J Physiol Gastrointest Liver Physiol 2006;290:G757-64.

39. Skogstrand K, Ekelund CK, Thorsen P, et al. Effects of blood sample handling procedures on measurable inflammatory markers in plasma, serum and dried blood spot samples. J Immunol Methods 2008;336:78-84.

40. Bell MJ, Ternberg JL, Feigin RD, et al. Neonatal necrotizing enterocolitis. Therapeutic decisions based upon clinical staging. Ann Surg 1978;187:1-7. 